\section{ENT CLINICAL ACTIVITY DURING COVID19 PANDEMIC}

Sevasti Konstantinidou, Nikita Mehtani, Claire Frauenfelder, Christopher Jephson. Great Ormond Street Hospital

\subsection{6/archdischild-2020-gosh.7}

Background During COVID19 pandemic all ENT registrars and SHOs were re-deployed to other specialties and senior clinical fellows were upgraded to consultants. The aim of this project is to analyse ENT clinical activity during the COVID19 pandemic in order to assess appropriateness of ENT staffing level and contribute in preparation for future similar events.

Methods Retrospective data collection was performed via Epic from 23/3/20 until 21/6/20. ENT admission and procedural data were collected and compared to other paediatric surgical subspecialties. Finally, a case study has been incorporated into our presentation to demonstrate the additional difficulties encountered in the care of complex airway patients during the COVID19 pandemic.

Results 143 patients were admitted under ENT. In total, 220 ENT operations were performed on ENT patients as well as patients admitted under other teams. $52.4 \%$ of these cases were airway procedures. When considering relative workload during this period, SNAPS performed 251 cases while ENT and Cardiothoracics both performed 220 operations.

The case report studied was an eight months girl with tracheal stenosis who required multiple admissions due to acute airway compromise. This case demonstrated that adversities encountered during the COVID19 pandemic included difficult access to local hospitals, challenging patient transfer, limited medical staff and theatre resources.

Conclusion During the COVID19 pandemic ENT continued to have a high workload of complex patients, especially relating to airway issues. This was on a background of restricted access to paediatric inpatient facilities and resources at other hospitals in North Central London. Following the re-deployment of all ENT junior doctors, ENT at GOSH became a consultant only service whilst SNAPS and cardiac surgery kept their full complement of juniors. The results of this study have helped in planning for a potential COVID19 second wave response, particularly around operating theatre allocation and in surgical junior workforce deployment.

\section{GREAT ORMOND STREET HOSPITAL (GOSH) SUMMER SCHOOL 2020: LESSONS ON DESIGNING A DIGITAL CONFERENCE}

${ }^{1}$ Shuang Liu, 'Jonathan Smith, ${ }^{2}$ Abbie Tutt, ${ }^{1}$ Justin Poisson, ${ }^{1}$ Simon Blackburn, ${ }^{1}$ Marissa Willock, 'Jeroen Poisson, 'Nicola Palmer, 'Shereen Brown, 'livia Wheeler, ${ }^{1}$ Peter Stow, ${ }^{1}$ Ellie McGonegal, ${ }^{1}$ Cristine Sortica da Costa. ${ }^{1} P G M E$, Great Ormond Street Hospital; ${ }^{2}$ Warwick University

10.1136/archdischild-2020-gosh.8

Background GOSH Summer School (GSS) is a conference for medical students and early career trainees. It has been run as a face-to-face event in the previous two years. COVID-19 outbreak challenged us to reformat the conference digitally.

Methods The GSS was run over four days, and the programme had main themes on each day: General Paediatric, Surgery and Innovation, Global health and COVID, and Paediatric Sub-specialties. The conference was hosted as webinar/ Q\&A sessions; questions were submitted via a digital platform with anonymous options. Participants were encouraged to interact via conference App.

Results $60 \%$ of the registrations were medical students from year 4 and 5. 630 different users logged in to the conference, with an average of 300 participants per session compared to $50-75$, in the previous two years. According to the daily polls, GSS was the first virtual conference for $59 \%$ of the participants since the pandemic; $58 \%$ preferred to ask questions using an online written platform, $4 \%$ preferred to speak directly, and $38 \%$ chose both. About the most productive method to deliver education, 36\% chose face-to-face sessions, $34 \%$ Webinars, and 22\% felt these two methods were considered equal. $78 \%$ voted that our format of 30 minutes presentation with 15 minutes Q \&A was 'about right.' 69\% felt our current format of 4-5 hours per day of webinar time was considered the best option. About 227 participants joined the conference App. 346 participants completed the post-conference survey, and 100\% voted to continue the online format for next year's conference.

Discussion The online GSS was as successful as the previous face-to-face events. We have learned that digital platforms can efficiently deliver high-quality education, promote interaction and engagement with the participants, and reach a larger number of students.

\section{REMOTE WOUND REVIEWS: EMPOWERING PATIENTS AND PARENTS THROUGHOUT COVID AND BEYOND}

Henry Bowyer, Sara Omar, James Yeats, Simon Humphry, Deborah Eastwood. Great Ormond Street Hospital

\subsection{6/archdischild-2020-gosh.9}

Background Post-operative wound reviews constitute an essential part of care for children undergoing surgery, allowing prompt identification of post-operative complications.

Until now it has been routine practice in the orthopaedic department to delegate wound reviews to community services. However, departmental follow-up may have significant benefits including improved continuity of care. Additionally COVID-19 has meant that avoiding face-to-face consultations has greater appeal, particularly for patients with significant co-morbidities.

We developed a remote service for safe and patient-centred orthopaedic wound checks.

Methods Stake-holder perspectives were captured by surveying parents as well as orthopaedic surgeons within the department. Suitable patients were identified and provided with a choice between remote wound checks (RWC's) versus community services as standard. Patients opting for RWC's were asked to upload photographs of their wounds to MyGOSH two weeks post discharge. Once images had been reviewed a telephone consultation took place during which the Bluebelle wound healing questionnaire was carried out. Parental satisfaction scores were gathered following telephone consultation.

Results $100 \%$ of staff respondents supported a RWC service. A total of 7 patients were identified as suitable for this pilot, all opted for RWC over routine community follow-up.

All 7 patients received a remote wound check within 2 weeks of discharge (mean 11.7 days). One parent reported visiting their GP prior to remote review citing low confidence with dressing removal. All patients had reassuring photos and Bluebelle scores requiring no further follow-up prior to routine clinic. The median Bluebelle score for the cohort was 3 